\title{
User Mobility Modeling and Characterization of Mobility Patterns
}

\author{
Mahmood M. Zonoozi and Prem Dassanayake, Member, IEEE
}

\begin{abstract}
A mathematical formulation is developed for systematic tracking of the random movement of a mobile station in a cellular environment. It incorporates mobility parameters under the most generalized conditions, so that the model can be tailored to be applicable in most cellular environments. This mobility model is used to characterize different mobility-related traffic parameters in cellular systems. These include the distribution of the cell residence time of both new and handover calls, channel holding time, and the average number of handovers. It is shown that the cell resistance time can be described by the generalized gamma distribution. It is also shown that the negative exponential distribution is a good approximation for describing the channel holding time.
\end{abstract}

Index Terms-Mobile communication.

\section{INTRODUCTION}

$\mathbf{T}$ HE MOBILITY model plays an important role in examining different issues involved in a cellular system such as handover, offered traffic, dimensioning of signaling network, user location updating, registration, paging, multilayer network management, and the like. In the general case, the mobility modeling should include changes in both the direction and speed of the mobile. Since the moving direction and the speed of a mobile are both nondeterministic variables, the path of a mobile will be a random trajectory. Tracing this trajectory requires a systematic formulation of the geometrical relations governing the complex problem of random movement. A review of the available literature on this subject reveals that only a few of the works have dealt with the related matter in detail, although the need for a comprehensive study is evident. Mobility models developed in the literature [1], [2] assume constant speeds drawn from given probability distributions. Guérin [3] has developed a mobility model where the direction of a mobile is allowed to change at certain points in time. Tekinay [4] has proposed an approach based on the twodimensional random walk where the users are uniformly distributed in the area. This characteristizes the mobile movement as a modified Brownian motion. Some of the works consider mobility models for specific application purposes, such as grid patterns for two-dimensional space [5] and highway patterns for one-dimensional space [6]. The mobility model proposed in this paper considers all of the possible mobility-related

Manuscript received September 1, 1996; revised April 1, 1997. This work was supported by the Australian Telecommunications and Electronics Research Board (ATERB). The work of M. M. Zonoozi was supported by the Telecommunication Company of Iran.

The authors are with the Department of Electrical and Electronic Engineering, Victoria University of Technology, Melbourne, Vic. 8001, Australia.

Publisher Item Identifier S 0733-8716(97)05844-7. parameters including: mobile origin attributes (initial position, direction, and speed), ongoing attributes (changes in position, direction, and speed), and mobile destination attributes (final position, direction, and speed). This model is used to obtain different mobility-related traffic parameters.

Guérin [3] has shown that the channel holding time follows a negative exponential distribution. Morales-Andres et al. [7] and Thomas et al. [8] have used a fluid-flow model of mobility, and have analytically formulated the cell boundary crossing rate. Pollini et al. [9] have used these results to calculate the amount of signaling information needed to deliver calls to mobile stations. Seskar et al. [10] have shown via simulation that while the model given in [8] provides a good estimate of the boundary crossing rates for a Manhattan grid of sheets, other conditions lead to crossing rates larger or smaller than those of the model. Nanda [11] and Hong et al. [1] have analyzed the mean handover rate. Among the different mobility-related traffic parameters, one that has not received sufficient attention so far is the user's cell residence time. Therefore, an appropriate probability distribution that accurately describes the cell residence time remains an issue to be investigated. A literature survey shows that a relatively few in-depth papers have been published on this subject, and most of these are restricted to simple mobility situations.

Hong and Rappaport [1] have made an elaborate analysis to obtain the cell residence time probability density function (pdf) for a simplified case of mobility in which there is no change in speed or direction of the mobile. Further, in this work, the initial speed of the mobile was assumed to follow a uniform distribution. Del Re et al. [2] have assumed that mobiles, before crossing a cell, travel a distance uniformly distributed between 0 and $2 R$, where $R$ is the hexagonal cell side. They also assume a constant speed with uniform distribution and conclude that the pdf of cell residence time is different from that shown in [1]. Inoue et al. [12] have applied the procedure of [1] for the case of nonuniform speed distribution. However, they end up with a set of unsolved integral equations. Yeung and Nanda [13], [14], Xie and Kuek [15], and Xie and Goodman [16] have shown that, contrary to the assumption made in [1], the speed and direction distributions of the in-cell mobiles are different from those of the cell-crossing mobiles. A more precise distribution for the speed and direction can be obtained using their biased sampling formula.

While Sanchez Vargas [17] and Lue [18] have assumed cell residence time to be uniformly distributed over the call duration, Nanda [11] and Lin et al. [19] have taken a general distribution for the cell residence time. Malyan et al. [20] have 


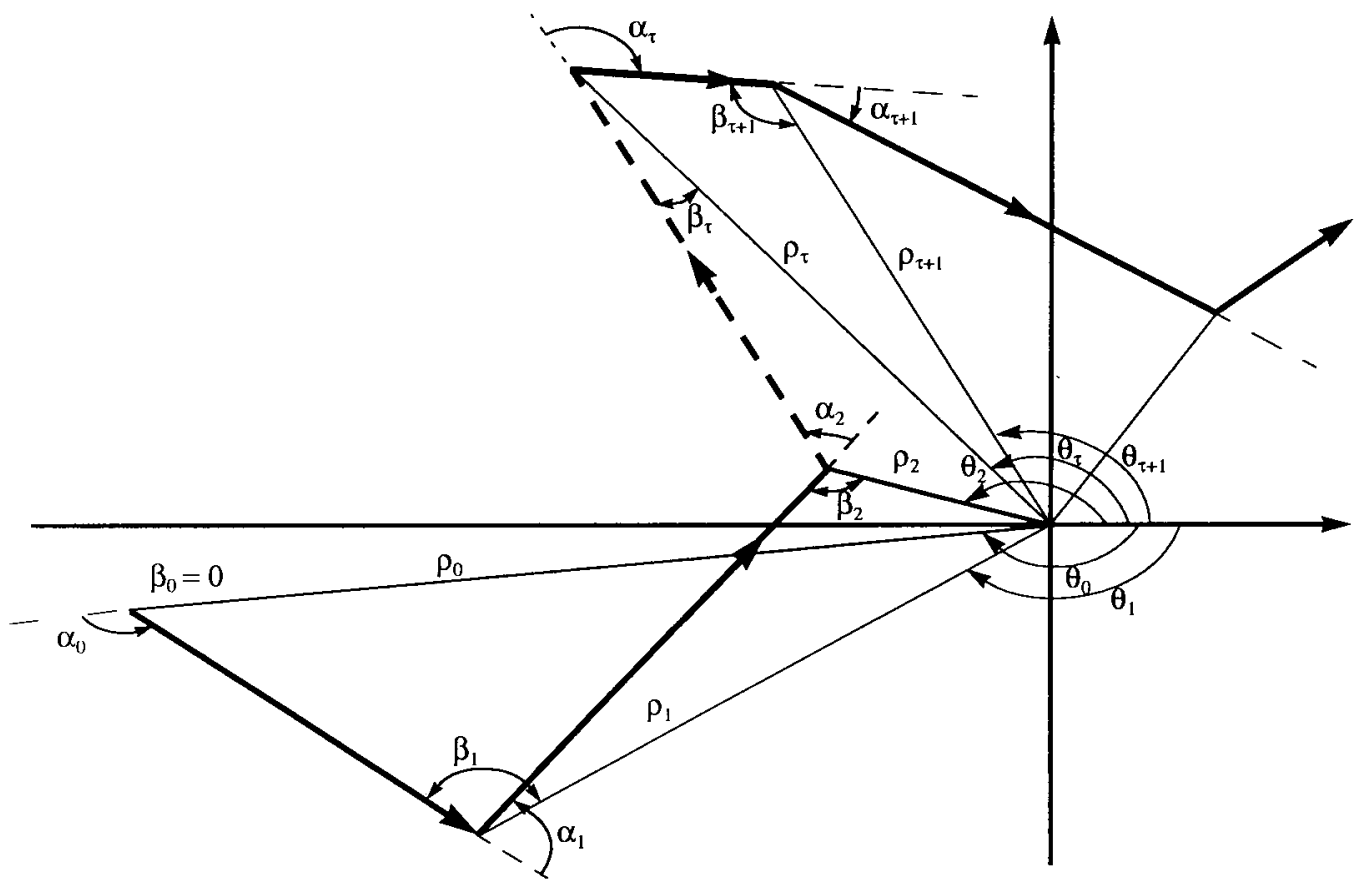

Fig. 1. Trajectory of a randomly moving mobile in the cellular environment.

proposed a model where a mobile is positioned initially at the center of a circular coverage area, and its cell residence time is obtained by using a two-dimensional random walk model. Generally, for the sake of simplicity, in the absence of any proven probability distribution, many authors dealing with the mobility problem have assumed, either explicitly or implicitly, the cell residence time to be an exponentially distributed random variable [21]-[28].

Another important parameter that appears in relation to cellular mobile systems is the channel holding (or occupancy) time. A knowledge of the channel holding time probability distribution function is necessary to obtain an accurate analysis of many teletraffic issues that arise in the planning and design of cellular mobile radio systems. The channel holding time of a cell is defined as the time during which a new or handover call occupies a channel in the given cell, and it is dependent on the mobility of the user. While this is similar to the call holding time in the fixed telephone network, it is often a fraction of the total call duration in a cellular mobile network and need not have the same statistical properties. Negative exponential distributions have been assumed to describe the channel holding time in modeling large and single-cell systems [29]-[31]. Guérin [3] has extended this by attempting to describe the channel holding time by the negative exponential distribution.

The outline of this paper is as follows. A systematic tracking of the random movement of a mobile station in a cellular environment is formulated mathematically in Sections II and III. Based on this formulation, a computer simulation is developed to obtain the behavior of different mobility-related parameters. An analysis of data obtained by simulation is given in Section IV, which shows that the generalized gamma distribution function is a good approximation for the cell residence time distribution. Section V deals with the mean cell residence time. The effect of changes in direction and speed is analyzed, and empirical relationships that relate these changes to the cell size are derived in Section VI. In Section VII, an expression to determine the average number of handovers in a cell is derived. Section VIII shows that the channel holding time of a cellular network is a negative exponential distribution.

\section{Tracing of A Mobile Inside the Cell}

The trajectory of a mobile in the cellular environment is shown in Fig. 1. Let $\left(\rho_{\tau}, \theta_{\tau}\right)$ denote the position of a mobile at an instant $\tau$. The coordinates of the mobile at any instant of time can be determined from the following relations [32]:

$$
\begin{aligned}
& \rho_{\tau+1}=\sqrt{\rho_{\tau}^{2}+d^{2}+2 \rho_{\tau} d \cos \gamma_{\tau}} \\
& \theta_{\tau+1}=\theta_{\tau} \pm \alpha_{\tau} \pm \beta_{\tau} \pm \beta_{\tau+1}
\end{aligned}
$$

where $\gamma_{\tau}$ is the supplementary angle between the current direction of the mobile and the line connecting the previous position of the mobile to the base station, $\alpha_{\tau}$ is the change in direction at time $\tau$ with respect to the previous direction, $\beta_{\tau}$ is the magnitude of the angle between the mobile's previous direction and the line joining the mobile's current position to the reference point (base station), and $d$ is the distance traversed during the time interval $\Delta \tau$ between $\tau$ and $\tau+1$. If the mobile speed during $\Delta \tau$ is assumed to be $\nu$, then, $d=\nu \cdot \Delta \tau$. The signs + or - depend on the successive positioning of the mobile, and have to be ascertained as shown latter. In order to simplify the formulation, a coordinate system is defined with its origin at the current location of the mobile. In this coordinate system, the positive $x$ axis coincides with the mobile's previous moving direction, and the $y$ axis coincides with the line joining the current mobile location to the base station. The positive direction 


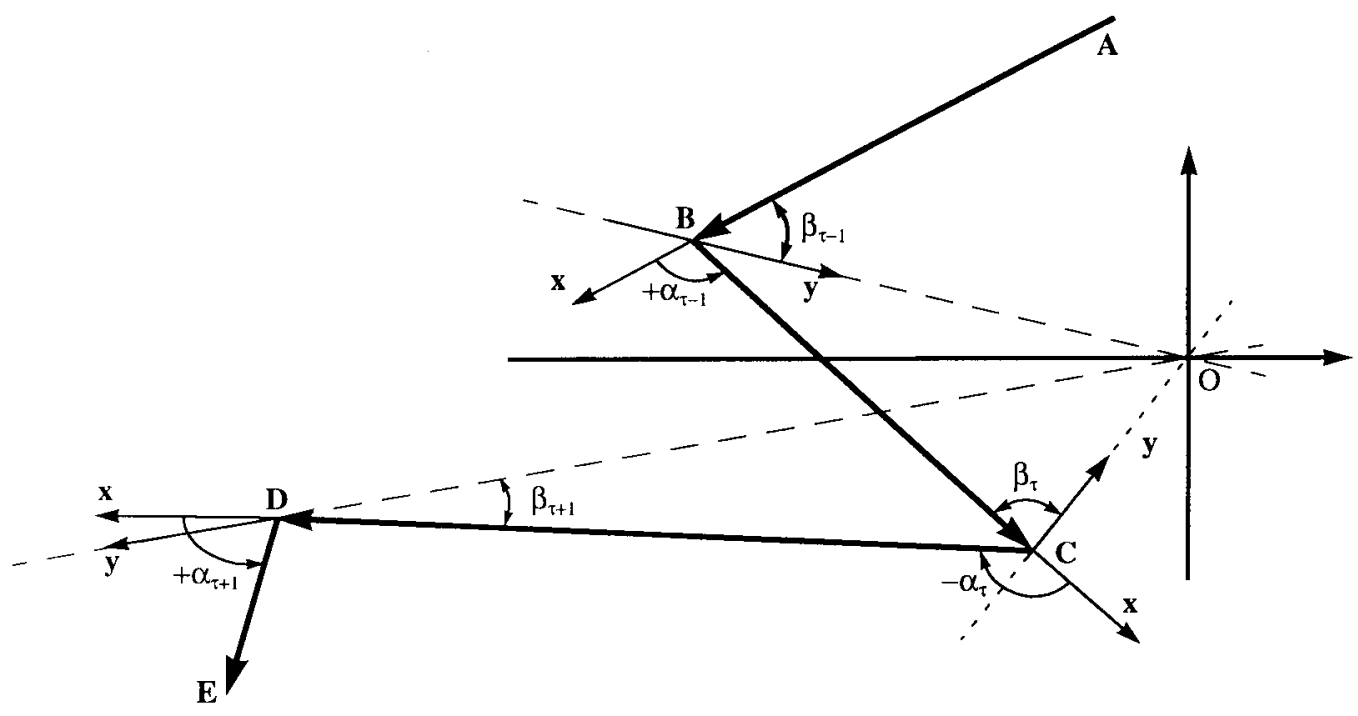

Fig. 2. An example of movement of a mobile from location $A$ to $E$ passing through the regions of $1(A B C), 2(C D)$, and $4(D E)$.
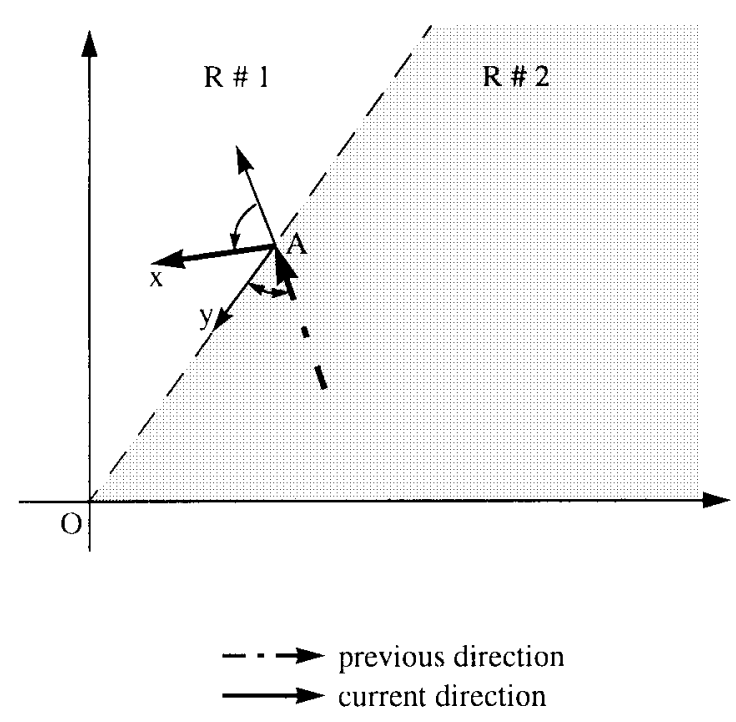

(a)

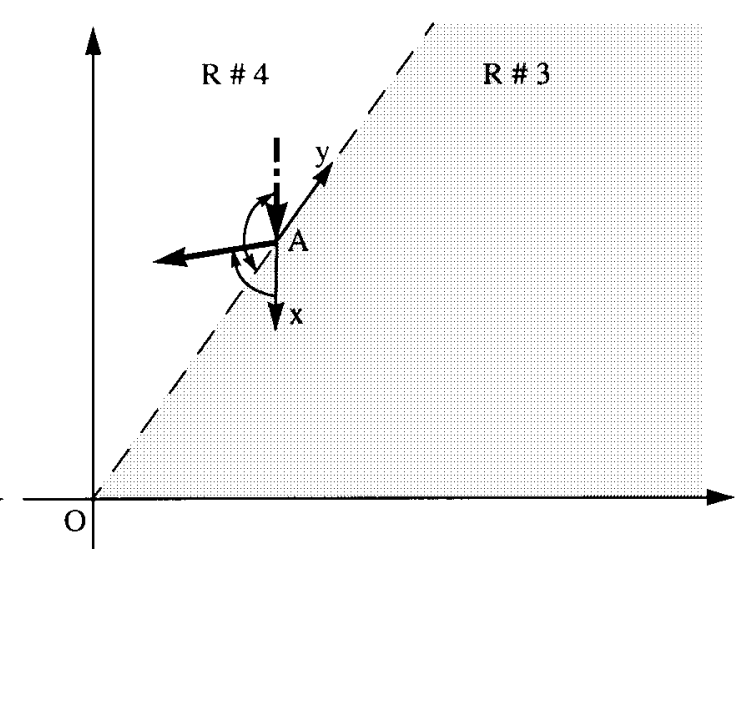

(b)

Fig. 3. Illustration of the different regions.

of the $y$ axis is obtained by turning counterclockwise from the $x$ axis until the $y$ axis is met. The angle between the two axes can be any value between 0 and $\pi$, depending on the previous direction of the mobile (Fig. 2). Such a coordinate system is dynamic in the sense that its origin and axes orientation change according to the successive locations and directions of the mobile. Further, it can be seen that the positive $y$ axis can be either toward or outwards from the base station, depending on the mobile movement direction.

Consider a mobile located at point $A$ at time $\tau$. If the mobile approaches the point $A$ as shown in Fig. 3(a), then the positive $y$ axis is toward the base station. However, if the mobile approaches the point $A$ as shown in Fig. 3(b), then the positive $y$ axis is outwards from the base station. Notice that the line joining the mobile to the base station $A O$ divides the cell space into two regions. If the positive $y$ axis at point $A$ is toward the base station, then $\alpha_{\tau}$ will relate to $\beta_{\tau}$ such that the two regions can be identified as

$$
\begin{array}{lrl}
\text { Region } 1(R 1): & -\beta_{\tau} \leq \alpha_{\tau} \leq \pi-\beta_{\tau} \\
\text { Region 2(R2): } & \alpha_{\tau}<-\beta_{\tau} \text { or } \alpha_{\tau}>\pi-\beta_{\tau} .
\end{array}
$$

Similarly, if the positive $y$ axis at point $A$ is outwards from the base station, then the two regions can be identified as

$$
\begin{array}{ll}
\text { Region } 3(R 3): & \beta_{\tau} \geq \alpha_{\tau} \geq \beta_{\tau}-\pi \\
\text { Region } 4(R 4): & \alpha_{\tau}>\beta_{\tau} \text { or } \alpha_{\tau}<\beta_{\tau}-\pi
\end{array}
$$

Since any value of $\alpha_{\tau}$ may satisfy one of (3) and one of (4), to determine $\alpha_{\tau}$ uniquely, we proceed as follows. Consider two successive points $E$ and $F$ in the mobile path (Fig. 4). Let $O E$ and $O F$ be the lines joining the base station to the points $E$ and $F$. Depending on the directions of the mobile at time 


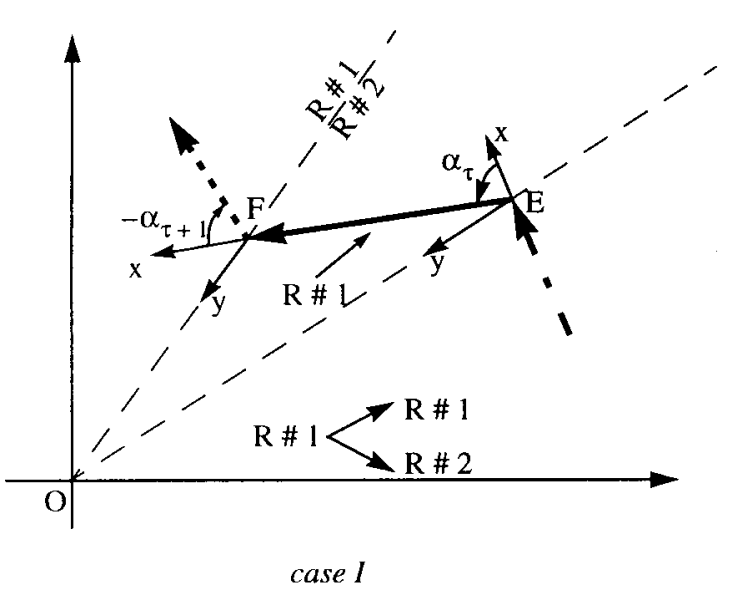

(a)

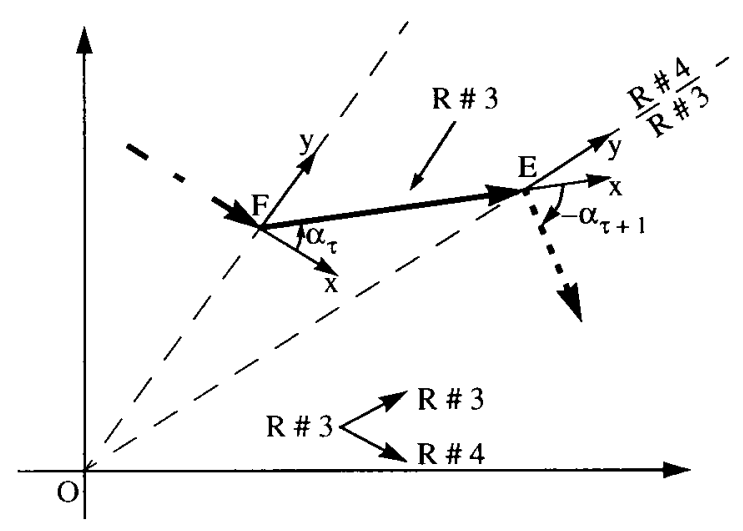

case III

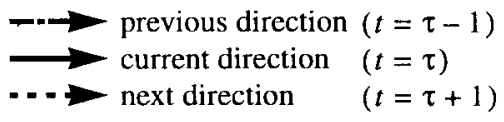

(c)

Fig. 4. Movement of mobiles between different regions.

$\tau$ and $\tau-1$, one of the following cases can occur:

$$
\begin{array}{ll}
\text { case I } & \left\{\begin{array}{l}
\text { direction } \Rightarrow \text { from } E \text { to } F \\
\text { last location } \Rightarrow \text { lower side of the line } O E
\end{array}\right. \\
\text { case II } \quad\left\{\begin{array}{l}
\text { direction } \Rightarrow \text { from } F \text { to } E \\
\text { last location } \Rightarrow \text { lower side of the line } O F
\end{array}\right. \\
\text { case III } \quad\left\{\begin{array}{l}
\text { direction } \Rightarrow \text { from } F \text { to } E \\
\text { last location } \Rightarrow \text { upper side of the line } O F
\end{array}\right. \\
\text { case IV }\left\{\begin{array}{l}
\text { direction } \Rightarrow \text { from } E \text { to } F \\
\text { last location } \Rightarrow \text { upper side of the line } O E .
\end{array}\right.
\end{array}
$$

Inspection of any of these cases reveals that the mobile movement is related to the regional transitions. That is, in case I, the mobile movement is such that it enters the region $R 1$ at point $E$. This mobile has the option of moving either to $R 1$ or to $R 2$ in continuing its movement at point $F$. As another example, consider case II. At point $E$, the mobile enters the

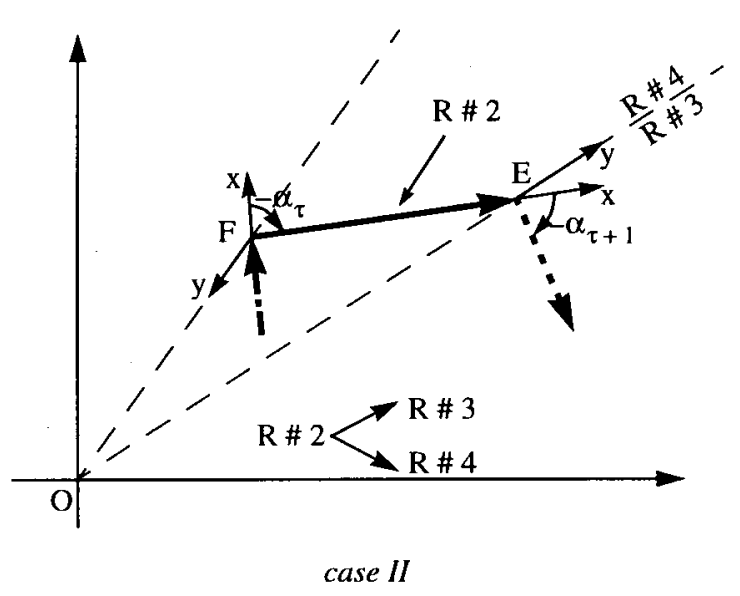

(b)

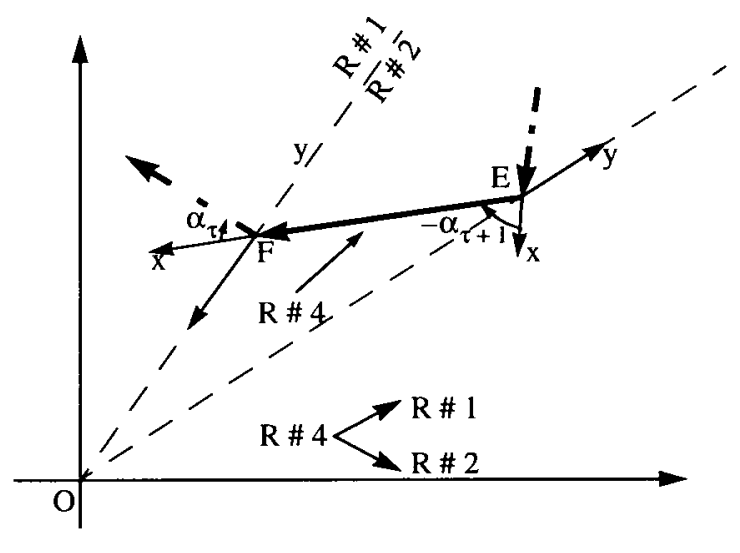

case IV

(d)

region $R 2$. At point $F$ it has the option of moving either to $R 3$ or to $R 4$. The same argument could be put in cases III and IV. These can be summarized as

case $\mathrm{I} \Rightarrow$ a mobile moving in $R 1$ will continue its motion either in $R 1$ or $R 2$

case II $\Rightarrow$ a mobile moving in $R 2$ will continue its motion either in $R 3$ or $R 4$

case III $\Rightarrow$ a mobile moving in $R 3$ will continue its motion either in $R 3$ or $R 4$

case IV $\Rightarrow$ a mobile moving in $R 4$ will continue its motion either in $R 1$ or $R 2$.

Examination of the successive positions of the mobile reveals that the mobile movement could be tagged to the regional transitions. For instance, a mobile in region $R 1$ at time $\tau$ can remain in region $R 1$ or move to region $R 2$ at time $\tau+1$. A mobile in region $R 2$ at time $\tau$ can move to 


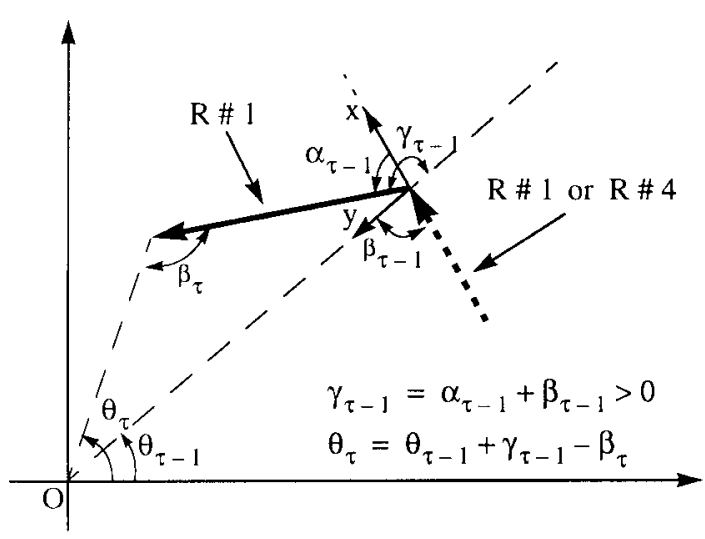

(a)

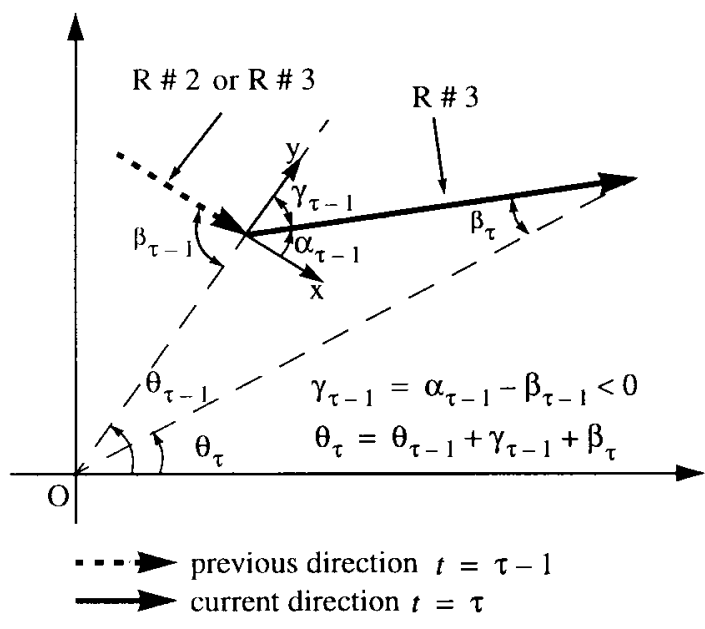

(c)

Fig. 5. Evaluation of $\theta_{\tau}$ in different regional transitions.

either region $R 3$ or region $R 4$ at time $\tau+1$, and so on. The value of $\theta_{\tau}$ depends on the current and the previous states of the mobile. For instance, if the mobile arrives at time $\tau$ to region $R 1$ (either from region $R 1$ or region $R 4$ ), $\theta_{\tau}$ is given by $\theta_{\tau}=\theta_{\tau-1}+\gamma_{\tau-1}-\beta_{\tau}$, where $\gamma_{\tau-1}=\alpha_{\tau-1}+\beta_{\tau-1}$. Further, the condition for the arrival at region $R 1$ is given by $\gamma_{\tau-1}>0$. Similarly, the condition for the arrival at other states and the corresponding expressions for $\theta_{\tau}$ can be found according to Fig. 5, and can be tabulated as shown in Table I.

\section{Tracing of Mobile Outside the Cell}

In order to follow the trajectory of a mobile moving outside a cell, it is necessary to trace it as it moves to adjacent cells, and this is described in [32].

\section{Cell Residence Time Distribution}

Depending on whether a call is originated in a cell or handed over from a neighboring cell, two different cell residence times can be specified. They are the new call cell residence time and the handover call cell residence time, respectively. The new call cell residence time is defined as the length of time a mobile terminal resides in the cell where the call originated before crossing the cell boundary. Similarly, the handover call

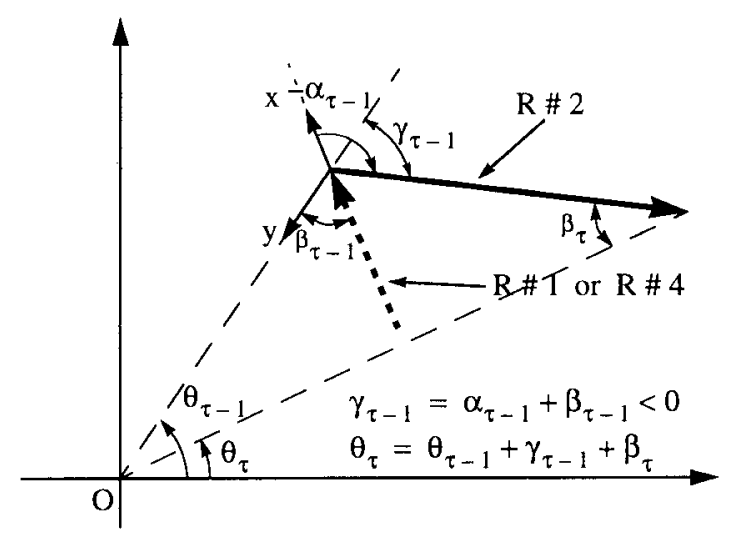

(b)

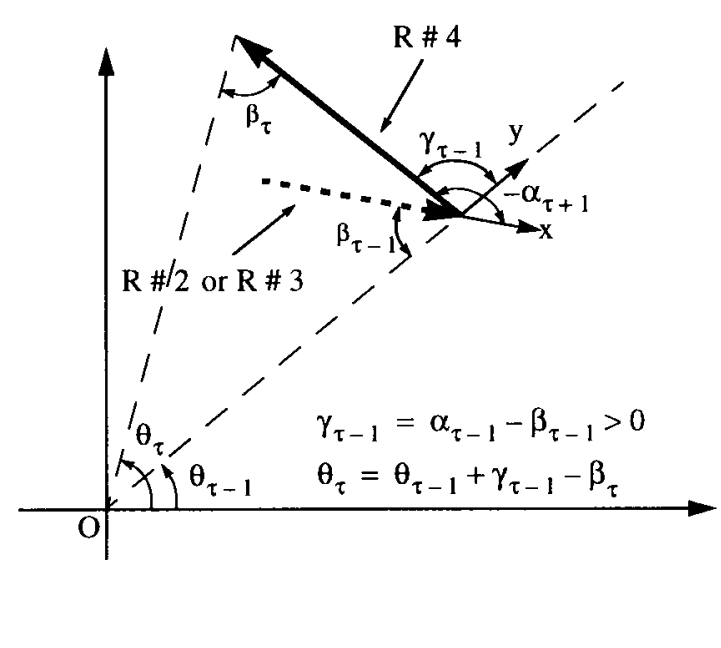

(d)

TABLE I

Equations for Calculation of Mobile New Location

\begin{tabular}{c|c}
\hline State Changes & Equations \\
\hline \hline $1 \Rightarrow 1$ or $4 \Rightarrow 1$ & $\begin{array}{c}\gamma_{\tau-1}=\alpha_{\tau-1}+\beta_{\tau-1}>0 \\
\theta_{\tau}=\theta_{\tau-1}+\gamma_{\tau-1}-\beta_{\tau}\end{array}$ \\
\hline $1 \Rightarrow 2$ or $4 \Rightarrow 2$ & $\begin{array}{c}\gamma_{\tau-1}=\alpha_{\tau-1}+\beta_{\tau-1}<0 \\
\theta_{\tau}=\theta_{\tau-1}+\gamma_{\tau-1}+\beta_{\tau}\end{array}$ \\
\hline $2 \Rightarrow 3$ or $3 \Rightarrow 3$ & $\begin{array}{c}\gamma_{\tau-1}=\alpha_{\tau-1}-\beta_{\tau-1}<0 \\
\theta_{\tau}=\theta_{\tau-1}+\gamma_{\tau-1}+\beta_{\tau}\end{array}$ \\
\hline $2 \Rightarrow 4$ or $3 \Rightarrow 4$ & $\begin{array}{c}\gamma_{\tau-1}=\alpha_{\tau-1}-\beta_{\tau-1}>0 \\
\theta_{\tau}=\theta_{\tau-1}+\gamma_{\tau-1}-\beta_{\tau}\end{array}$ \\
\hline
\end{tabular}

cell residence time is defined as the time spent by a mobile in a given cell to which the call was handed over from a neighboring cell before crossing to another cell. The new call cell residence time $T_{n}$ and the handover call cell residence time $T_{h}$ are two random variables whose distributions have to be found. The term "cell residence time" is also labeled as 


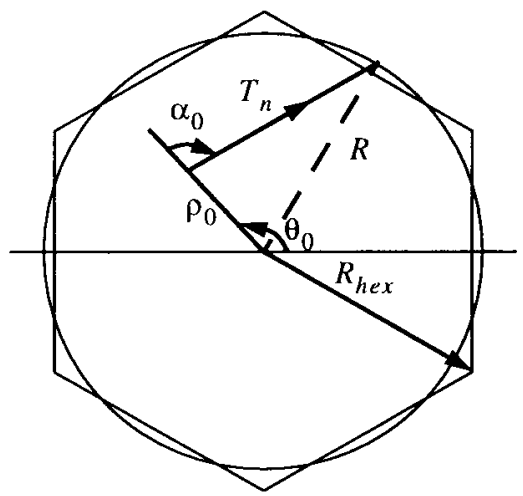

(a)

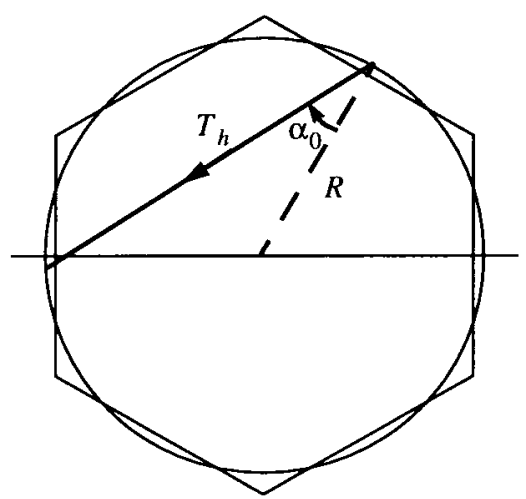

(b)

Fig. 6. Cell residence time illustration for two different cases. (a) New call $T_{n}$. (b) Handover call $T_{h}$.

the mobile sojourn time, dwell time or block holding time by some authors [14], [33], [34].

\section{A. Simplified Case}

Let $f_{T_{n}}(t)$ and $f_{T_{h}}(t)$ denote the probability density of the new call and handover call cell residence times, respectively. These probability functions can be obtained by [1] (Fig. 6)

$$
\begin{aligned}
& f_{T_{n}}(t)=\left\{\begin{array}{c}
\frac{8 R}{3 \pi V_{m} t^{2}}\left\{1-\left[1-\left(\frac{V_{m} t}{2 R}\right)^{2}\right]^{3 / 2}\right. \\
0 \leq t \leq \frac{2 R}{V_{m}} \\
\frac{8 R}{3 \pi V_{m} t^{2}}, \quad t \geq \frac{2 R}{V_{m}} \\
f_{T_{h}}(t)=\left\{\begin{array}{cc}
\frac{4 R}{\pi V_{m} t^{2}}\left\{1-\left[1-\left(\frac{V_{m} t}{2 R}\right)^{2}\right]^{1 / 2}\right.
\end{array}\right\} \\
\frac{4 R}{\pi V_{m} t^{2}}, \quad t \geq \frac{2 R}{V_{m}}
\end{array}\right.
\end{aligned}
$$

In [15] and [16], it is shown that the speed and direction distributions of the in-cell mobiles are different from those of the cell-boundary crossing mobiles. Let $f_{V_{0}}\left(\nu_{0}\right)$ denote the pdf of the speeds of the in-cell mobiles, and let $f_{V_{0}}^{*}\left(\nu_{0}\right)$ denote the pdf of the speeds of the cell-boundary crossing mobiles. Based on the biased sampling [35], it can be shown that

$$
f_{V_{0}}^{*}\left(\nu_{0}\right)=\frac{\nu_{0} f_{V_{0}}\left(\nu_{0}\right)}{E\left[V_{0}\right]}= \begin{cases}\frac{\nu_{0}}{V_{m} E\left[V_{0}\right]}, & 0 \leq \nu_{0} \leq V_{m} \\ 0, & \text { otherwise }\end{cases}
$$

where $E\left[V_{0}\right]$ is the mean speed. Similarly, let $f\left(\alpha_{0}\right)$ be the pdf of the directions of all mobile stations, which is uniform in the range $(0,2 \pi)$. Based on the biased sampling, the pdf of the directions of the cell-boundary crossing terminals $f^{*}\left(\alpha_{0}\right)$ can be obtained as

$$
f^{*}\left(\alpha_{0}\right)=\left\{\begin{array}{lc}
\frac{1}{2} \cos \left(\alpha_{0}\right), & -\frac{\pi}{2} \leq \alpha_{0} \leq \frac{\pi}{2} \\
0, & \text { otherwise. }
\end{array}\right.
$$

Considering (9) and (10), the relations for $f_{T_{n}}(t), F_{T_{n}}(t)$, $f_{T_{h}}(t)$, and $F_{T_{h}}(t)$ can be modified accordingly.

\section{B. Generalized Case}

Equations (7)-(8) represent the new and handover call cell residence time distributions for the simplified case of mobility in which there is no change in the speed and direction of the mobile, and there is no biasing in the speed or direction of the boundary crossing mobiles. In a general case, the mobility modeling should include changes in the direction and speed of the mobile. Moreover, it is unrealistic to assume that the speed is uniformly distributed and remains constant. It is virtually impossible to extend the analysis of the simplified case to cover the general case of mobility, and simulation appears to be the only way out. Based on the mobility model developed in Sections II and III, a computer simulation can be developed to study the mobility under generalized assumptions for different mobility-related parameters. Assumptions include: 1) users are independent and uniformly distributed over the entire region, 2) mobiles are allowed to move away from the starting point in any direction with equal probability, 3) the probability of the variation of the mobile direction (i.e., drift) along its path is taken to be a uniform distribution limited in the range of $\pm \alpha$ with respect to the current direction, 4) the initial velocity of the mobile stations is assumed to be a random variable with Gaussian probability density function truncated in the range $\left[V_{\min },=0, V_{\max }=100 \mathrm{~km} / \mathrm{h}\right]$, and 5) the velocity increment of each mobile is taken to be a uniformly distributed random variable in the range $\pm 10 \%$ of the current velocity.

What is of importance here is not the actual mobile trajectories, but the distribution of the users' cell residence time. With this in mind, we wish to test the hypothesis tht the new call and handover call residence time data follow a particular probability distribution. Following [36] and [37], we proceed with the generalized gamma distribution which provides probability density functions of the form

$$
f_{T}(t ; a, b, c)=\frac{c}{b^{a c} \Gamma(a)} t^{a c-1} e^{-(t / b)^{c}}, \quad t, a, b, c>0
$$

where $\Gamma(a)$ is the gamma function, defined as $\Gamma(a)=$ $\int_{0}^{\infty}\left(x^{a-1}\right) e^{-x} d x$ for any real and positive number $a$. The evaluation of the agreement between the distributions obtained by simulation and the best-fit generalized gamma distribution 


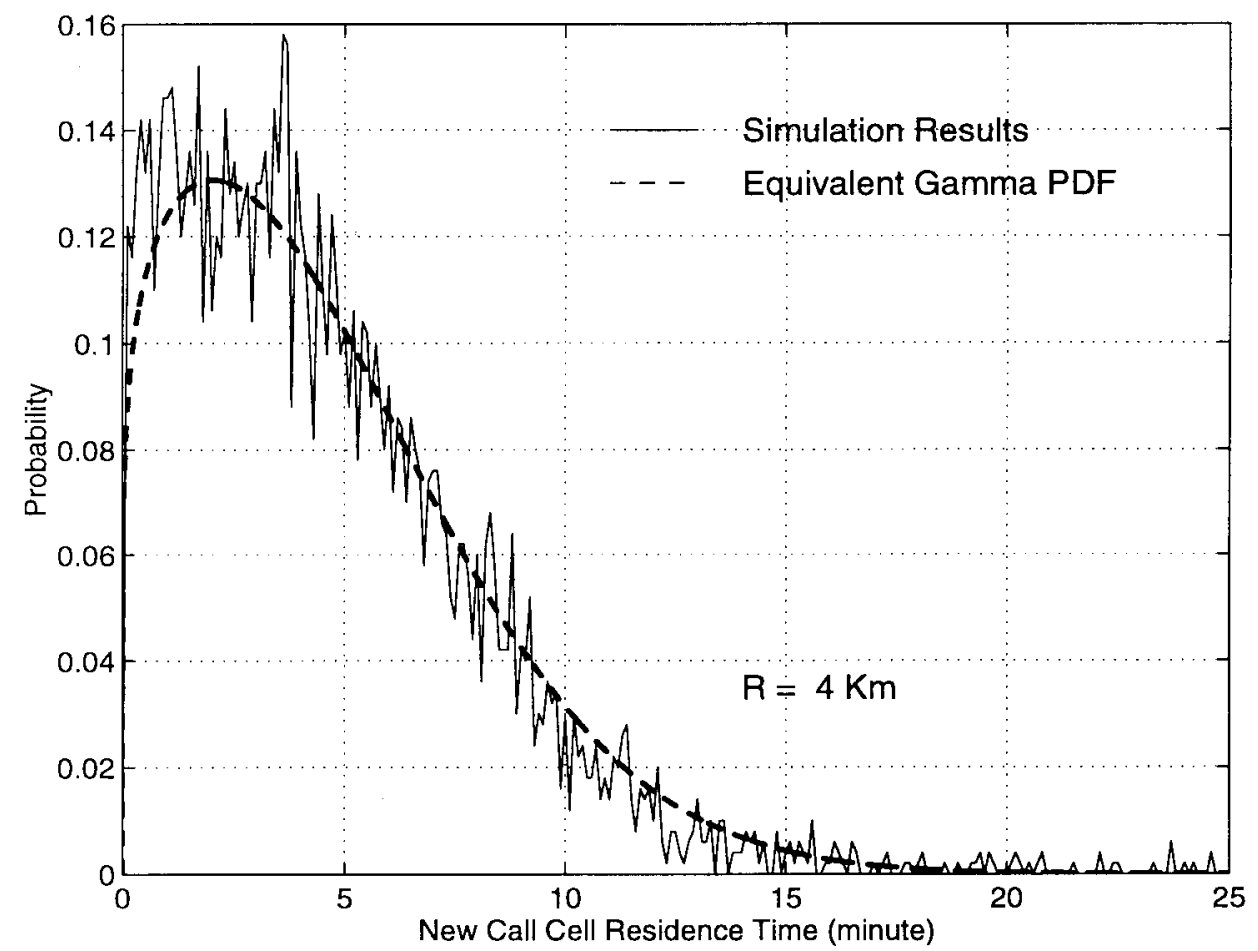

Fig. 7. New call cell residence time pdf.

is done by using the Kolmogorov-Smirnov goodness-offit test. Given the generalized gamma distribution as the hypothesized distribution, the values of the parameters $a, b, c$ are found such that the maximum deviation is a minimum. The maximum deviation shows the biggest divergence between the observed and the hypothesized distributions. An examination of the data for the case of mobiles with an average speed of $50 \mathrm{~km} / \mathrm{h}$ and zero drift shows the values of $a, b, c$ for the new call and the handover call cell residence times with a level of significance of 0.05 as follows:

$$
\begin{aligned}
a & = \begin{cases}0.62, & \text { new call } \\
2.31, & \text { handover call }\end{cases} \\
b & \approx \begin{cases}1.84 R, & \text { new call } \\
1.22 R, & \text { handover call }\end{cases} \\
c & = \begin{cases}1.88, & \text { new call } \\
1.72, & \text { handover call. }\end{cases}
\end{aligned}
$$

The data represent the case of mobiles with an average speed of $50 \mathrm{~km} / \mathrm{h}$ and zero drift. Fig. 7 illustrates the probability density function of the new call cell residence time, obtained by simulation, and the equivalent generalized gamma function.

\section{Mean Cell Residence Time}

The mean cell residence time for the new call and the handover call can be found by the following relations:

$$
\begin{aligned}
& E\left[T_{n}\right]=\int_{0}^{\infty} t \cdot f_{T_{n}}(t) d t \\
& E\left[T_{h}\right]=\int_{0}^{\infty} t \cdot f_{T_{h}}(t) d t .
\end{aligned}
$$

Yeung and Nanda [13], [14], have shown that for an arbitrary speed pdf and zero drift, the mean cell residence time can be obtained by the following equations:

$$
\begin{aligned}
& E\left[T_{n}\right]=\frac{8 R \cdot E[1 / V]}{3 \pi} \\
& E\left[T_{h}\right]=\frac{\pi R}{2 \cdot E[V]}
\end{aligned}
$$

where $R$ is the cell radius and $V$ is the speed of the mobile in the cell. A comparison of the results obtained from (15)-(16) with (13)-(14) assuming generalized gamma pdf for $f_{T_{21}}(t)$ and $f_{T_{h}}(t)$ shows that the difference (error) is less than $0.05 \%$ in the case of new calls and $0.015 \%$ in the case of handover calls.

\section{EFFECT OF CHANGE IN DiRECTION AND SPEED}

Depending on the street structure, a mobile can move in different paths and may possess different speeds. The extent of a mobile's change in direction (drift) and change in speed are the two parameters that govern its mobility pattern. The effect of change in the direction and/or speed of mobiles can be considered as equivalent to a change in the average distance traveled or time spent in the cell before moving out. Thus, any increase in a mobile's drift can be treated as contributing to an effective increase in the cell radius. Similarly, any increase in speed of the mobile can be treated as contributing to a decrease in the cell residence time, which can be interpreted as an effective decrease in the cell size. Therefore, cells with a broad variety of mobility parameters can be replaced by an equivalent reference cell with an effective radius. A 

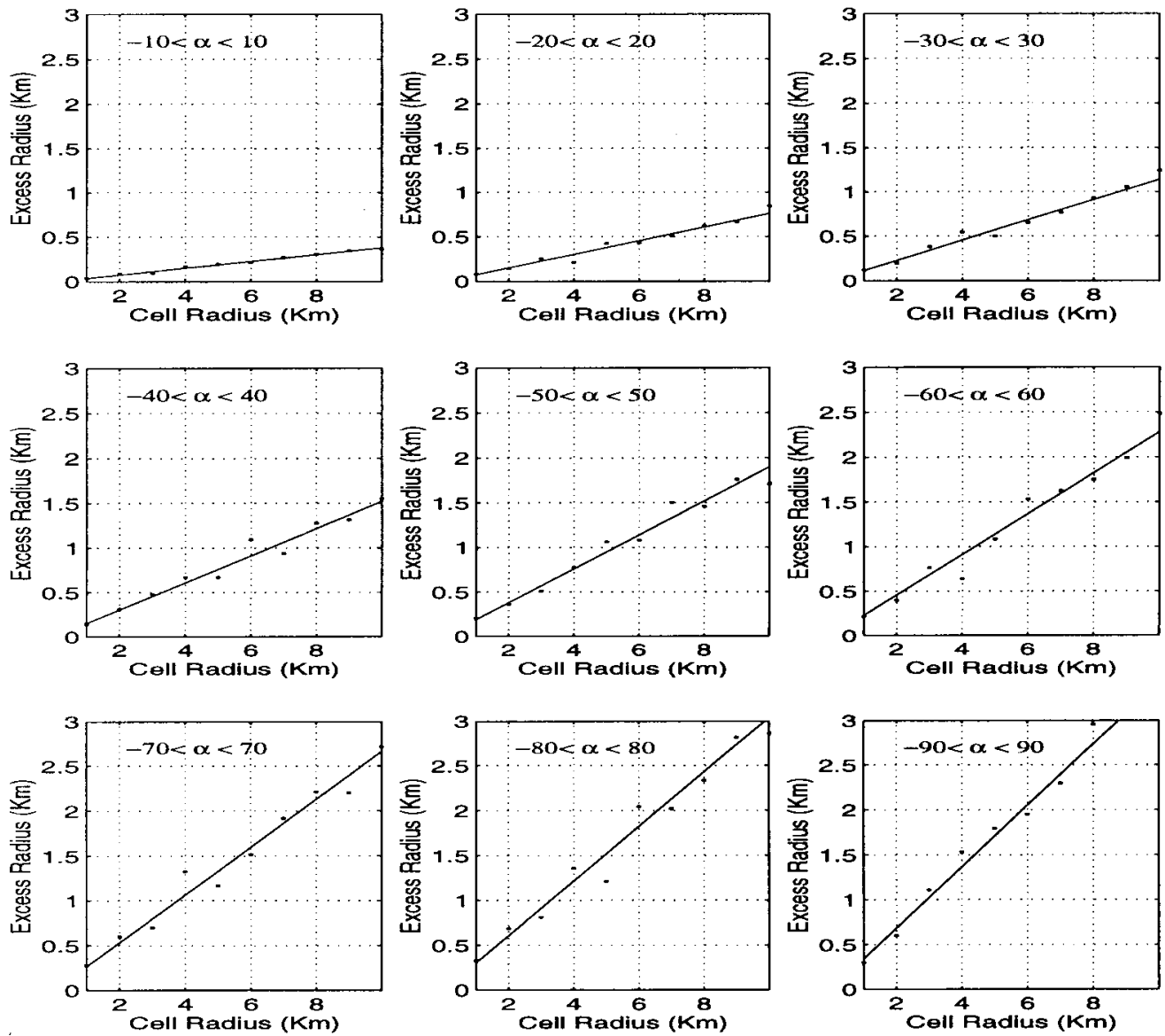

Fig. 8. Excess cell radius for different values of drift limits (in degrees).

reference cell is defined as a cell with the following mobility parameters.

1) The mobile moves in a straight path, i.e., $\alpha=0^{\circ}$.

2) The initial speed of a mobile follows a truncated Gaussian pdf with an average of $\mu_{\nu}=50 \mathrm{~km} / \mathrm{h}$ and standard deviation of $\sigma_{\nu}=15 \mathrm{~km} / \mathrm{h}$.

The main aim is to relate cells with given mobility parameters (i.e., drift $\alpha$ and average speed $\mu_{\nu}^{\prime}$ ) to the reference cell. Two different cases are considered.

Case 1) Cells in which mobiles move with a drift pdf in the range $-\varphi<\alpha<\varphi$ degrees and speed pdf similar to that of the reference cell. The radius of such cells is denoted by $R_{\alpha}$.

Case 2) Cells in which mobiles move with zero drift (similar to the reference cell) and a truncated Gaussian speed pdf with an average value of $\nu_{\nu}=\mu_{\nu}^{\prime} \mathrm{km} / \mathrm{h}$ and standard deviation of $\sigma_{\nu}=\left(\mu_{\nu}^{\prime}-5\right) / 3 \mathrm{~km} / \mathrm{h}$. The radius of such cells is denoted by $R_{\nu}$.

Consider a cell with the radius of $R_{\alpha}$ having mobility parameters according to Case 1). The radius of the equivalent cell $\Re_{\alpha}$ (which has the same residence time, but mobility parameters of the reference cell) is given by

$$
\Re_{\alpha}=R_{\alpha}+\Delta R_{\alpha}
$$

where $\Delta R_{\alpha}$ is the excess cell radius. Fig. 8 shows the excess cell radius for different drift limits. These curves allow handling of a variety of coverage areas with different street orientations and traffic flows by representing those with mobility equivalent cells with zero drift. The data obtained by simulation satisfy the empirical relation of (18) in a least mean-square sense

$$
\Delta R_{\alpha}=0.0038 \varphi R_{\alpha}
$$

Therefore, the equivalent cell radius will be

$$
\Re_{\alpha}=K_{\alpha} R_{\alpha}
$$

where $K_{\alpha}$ is the proportionality factor and is equal to $(0.0038 \varphi+1)$. In the same manner, consider a cell with the radius of $R_{\nu}$ and mobility parameters according to Case 2). The radius of an equivalent reference cell $\Re_{\nu}$, which has the same cell residence time but the mobility parameters of a reference cell, is given by

$$
\Re_{\nu}=R_{\nu}+\Delta R_{\nu}
$$

where $\Delta R_{\nu}$ is the excess cell radius. Fig. 9 shows the excess cell radius for different values of speed obtained by simulation. The data obtained by simulation satisfy the empirical equation (21) in a least mean-square sense

$$
\Delta R_{\nu}=\left(\frac{\mu_{\nu}}{\mu_{\nu}^{\prime}}-1\right) R_{\nu} .
$$




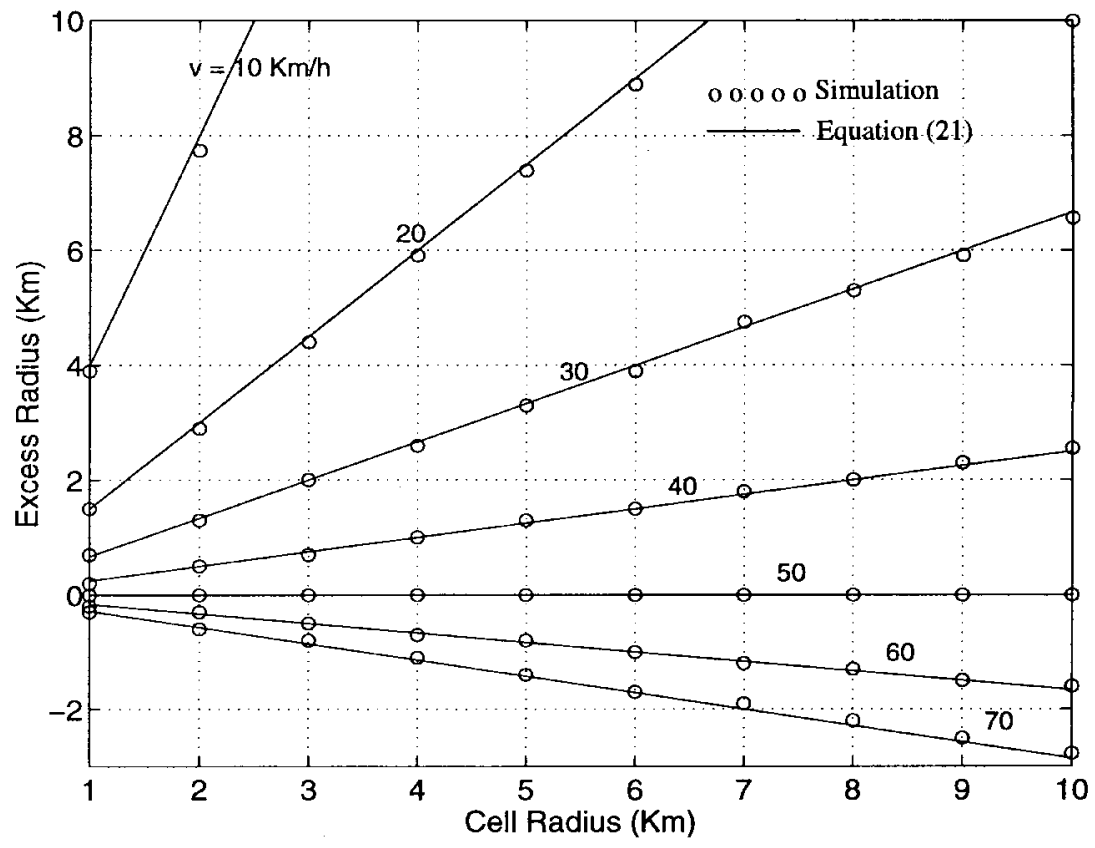

Fig. 9. Excess cell radius for different values of mean initial speed.

Considering $K_{\nu}=\mu_{\nu} / \mu_{\nu}^{\prime}$ as the proportionality factor, the equivalent cell radius will be

$$
\Re_{\nu}=K_{\nu} R_{\nu} .
$$

In a case where both drift and speed are different from those of the reference cell, the equivalent cell radius $\Re_{\alpha \nu}$ for a cell of radius $R_{\alpha \nu}$ can be obtained by the following relation $\left(R_{\alpha \nu}\right.$ is the cell radius of a cell which supports the mobility parameters of $\alpha$ and $\mu_{\nu}^{\prime}$ ):

$$
\Re_{\alpha \nu}=K_{\nu} K_{\alpha} R_{\alpha \nu} .
$$

Therefore, in a cell of radius $R_{\alpha \nu}$, the gamma distribution parameters $a, b, c$ for a mobile with an average speed $\mu_{\nu}^{\prime}$ and a drift $\left(-\varphi^{\circ}<\alpha<\varphi^{\circ}\right)$ can be described as per (12)

$$
\begin{aligned}
& a= \begin{cases}0.62, & \text { new call } \\
2.31, & \text { handover call }\end{cases} \\
& b \approx \begin{cases}1.84 \Re_{\alpha \nu}, & \text { new call } \\
1.22 \Re_{\alpha \nu}, & \text { handover call }\end{cases} \\
& c= \begin{cases}1.88, & \text { new call } \\
1.72, & \text { handover call. }\end{cases}
\end{aligned}
$$

\section{AVERAGe Number of HANDOvers}

A mobile can move through several cells while being involved in a call. The number of times a mobile crosses different boundaries during a call is a random variable dependent on the cell size, call holding time, and mobility parameters. Each handover requires network resources to reroute the call through a new base station. It is preferred to have as few handovers as possible in order to alleviate the switching load and to decrease the processing burden required in the system. The number of handovers has a lower bound which is equal to the number of boundary crossings a mobile undergoes. As the number of handovers increases, the handover decision algorithms need to be enhanced so that the perceived QoS does not deteriorate and the cellular infrastructure cost does not skyrocket. In the following subsections, we present two different methods to determine the average number of handovers in a cellular system.

\section{A. Method I}

The average number of times a nonblocked call is successfully handed over to the neighbor cell during the call can be obtained from

$$
E[H]=\sum_{k} k \cdot \operatorname{Prob}\{H=k\}
$$

where $\operatorname{Prob}\{H=k\}$ is the probability that a nonblocked call has $k$ successful handovers to the successive cells during its lifetime and $H$ is an integer random variable. Let $P_{n}$ be the probability that a nonblocked new call will require at least one handover before completion, let $P_{h}$ denote the probbility that a nonfailed handover call will require at least one more handover before completion, and let $P_{F h}$ be the probability that a handover attempt fails. Then [4]

$$
\begin{aligned}
\operatorname{Prob}\{H=0\}= & \left(1-P_{n}\right)+P_{n} P_{F h} \\
\operatorname{Prob}\{H=1\}= & P_{n}\left(1-P_{F h}\right)\left(1-P_{h}+P_{h} P_{F h}\right) \\
& \cdots \\
\operatorname{Prob}\{H=k\}= & P_{n}\left(1-P_{F h}\right)\left[P_{h}\left(1-P_{F h}\right)\right]^{k-1} \\
& \cdot\left(1-P_{h}+P_{h} P_{F h}\right) .
\end{aligned}
$$

Substituting (26) in (25) gives the average number of handovers per call $E[H]$ as the following:

$$
E[H]=\frac{P_{n}\left(1-P_{F h}\right)}{1-P_{h}\left(1-P_{F h}\right)} .
$$

Let random variables $T_{n}, T_{h}, T_{c}$ denote the new call residence time, the handover call residence time, and the call 


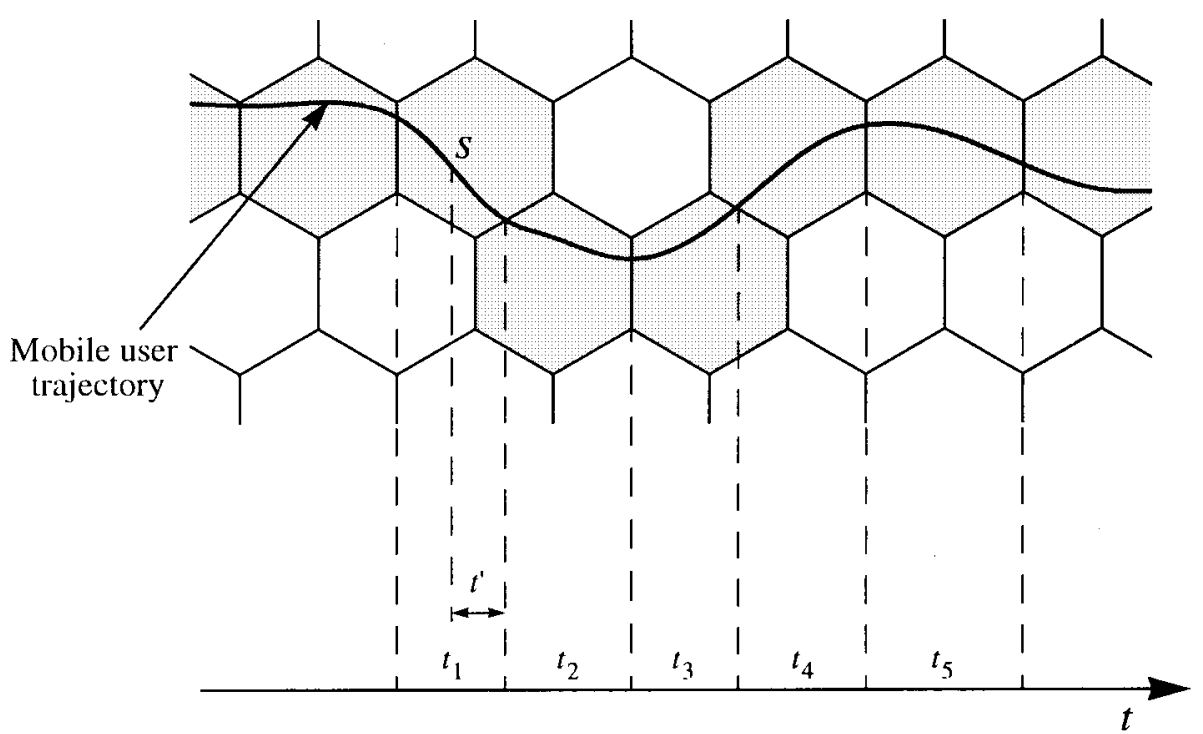

Fig. 10. Cell residence times for a mobile traveling across cells.

holding time, respectively. From classical teletraffic theories, it is well known that the call holding time follows a negative exponential distribution, i.e., the probability that any randomly selected call holding time will end in time duration $t$ is

$$
F_{T_{c}}(t)=1-e^{-\mu_{c} t}
$$

where $\bar{T}_{c}=1 / \mu_{c}$ is the average call holding time. The value of $P_{n}$ can be obtained by

$$
\begin{aligned}
P_{n} & =\operatorname{Prob}\left\{T_{c}>T_{n}\right\} \\
& =\int_{0}^{\infty} \operatorname{Prob}\left\{T_{c}>t \mid T_{n}=t\right\} \operatorname{Prob}\left\{T_{n}=t\right\} d t .
\end{aligned}
$$

$T_{n}$ is mainly dependent on the mobility of the users, and has no influence on the call duration $T_{c}$. Therefore

$$
\begin{aligned}
P_{n} & =\int_{0}^{\infty} \operatorname{Prob}\left\{T_{c}>t\right\} \cdot \operatorname{Prob}\left\{T_{n}=t\right\} d t \\
& =\int_{0}^{\infty}\left[1-F_{T_{c}}(t)\right] \cdot f_{T_{n}}(t) d t \\
& =\int_{0}^{\infty} e^{-\mu_{c} t} \cdot f_{T_{n}}(t) d t .
\end{aligned}
$$

Similarly, the value of $P_{h}$ can be obtained by the following equation:

$$
P_{h}=\operatorname{Prob}\left\{T_{c}>T_{h}\right\}=\int_{0}^{\infty} e^{-\mu_{c} t} \cdot f_{T_{h}}(t) d t .
$$

The values of $P_{n}$ and $P_{h}$ can be numerically evaluated by substituting a generalized gamma pdf for $f_{T_{n}}(t)$ and $f_{T_{h}}(t)$ in (30)-(31), respectively.

\section{B. Method II}

Consider a noise traversing a path with consecutive cell residence times $t_{1}, t_{2}, \cdots$ as in Fig. 10. Assume that a call starts at point $S$ so that the remaining cell residence time in the cell is $t^{\prime}$. In other words, the random variable $T_{h}=\left\{t_{1}, t_{2}, \cdots\right\}$ is the handover cal cell residence time, and the random variable $T_{n}=\left\{t^{\prime}\right\}$ is the new call cell residence time. The random variables $T_{h}$ and $T_{n}$ are assumed to be independent and identically distributed with general distributions of $f_{T_{h}}(t)$ and $f_{T_{n}}(t)$. The Laplace transform of the handover call cell residence time $f_{h}^{*}\left(\mu_{c}\right)$ can be defined as

$$
f_{h}^{*}\left(\mu_{c}\right)=\int_{0}^{\infty} f_{T_{h}}(t) e^{-\mu_{c} t} d t=E\left[e^{-\mu_{c} t}\right]
$$

From the excess life theorem [38] (or residual service time [39]), the Laplace transformation of the new call cell residence time $f_{n}^{*}\left(\mu_{c}\right)$ can be obtained as the following:

$$
f_{n}^{*}\left(\mu_{c}\right)=\frac{1}{\mu_{c} E\left[T_{h}\right]}\left[1-f_{h}^{*}\left(\mu_{c}\right)\right]
$$

The number of handovers experienced by a call depends on the call holding time (which is exponentially distributed with mean $1 / \mu_{c}$ ), and can be obtained as follows [11]:

$$
\begin{aligned}
E\left[H \mid t^{\prime}, t_{2}, t_{3}, \cdots\right] & \\
= & 1 \cdot \int_{t^{\prime}}^{t^{\prime}+t_{2}} \mu_{c} e^{-\mu_{c} x} d x+2 \cdot \int_{t^{\prime}+t_{2}}^{t^{\prime}+t_{2}+t_{3}} \mu_{c} e^{-\mu_{c} x} d x \\
& +3 \cdot \int_{t^{\prime}+t_{2}+t_{3}}^{t^{\prime}+t_{2}+t_{3}+t_{4}} \mu_{c} e^{-\mu_{c} x} d x+\cdots \\
= & \int_{t^{\prime}}^{\infty} \mu_{c} e^{-\mu_{c} x} d x+\int_{t^{\prime}+t_{2}}^{\infty} \mu_{c} e^{-\mu_{c} x} d x \\
& +\int_{t^{\prime}+t_{2}+t_{3}}^{\infty} \mu_{c} e^{-\mu_{c} x} d x+\cdots \\
= & e^{-\mu_{c} t^{\prime}}\left[1+e^{-\mu_{c} t_{2}}\left[1+e^{-\mu_{c} t_{3}}[1+\cdots .\right.\right.
\end{aligned}
$$

Considering $E\left[E\left[H \mid t^{\prime}, t_{2}, t_{3}, \cdots\right]\right]=E\left[H \mid t^{\prime}\right]$ and using (32), we rewrite (34) as follows:

$$
\begin{aligned}
E\left[H \mid t^{\prime}\right] & =e^{-\mu_{c} t^{\prime}}\left[1+f_{h}^{*}\left(\mu_{c}\right)\left[1+f_{h}^{*}\left(\mu_{c}\right)[1+\cdots\right.\right. \\
& =\frac{e^{-\mu_{c} t^{\prime}}}{1-f_{h}^{*}\left(\mu_{c}\right)} .
\end{aligned}
$$




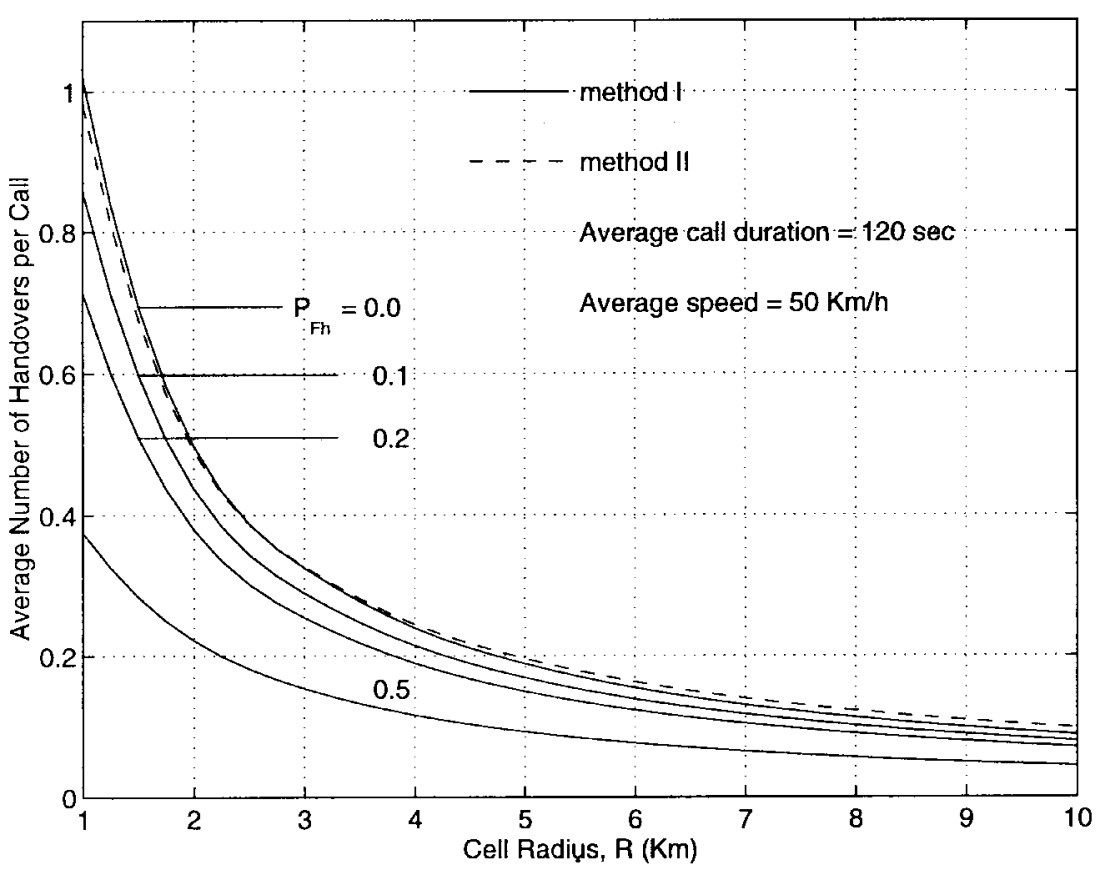

Fig. 11. Average number of handovers experienced by a call for different probabilities of handover failures $P_{F h}$.

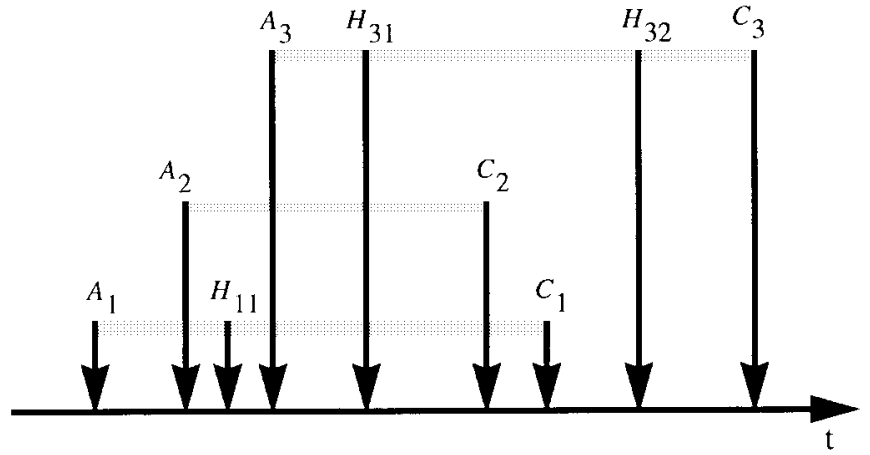

Fig. 12. Illustration of different types of handover within various call durations.

Taking the expectation of (35) and considering (32) and (33), we have

$$
\begin{aligned}
E[H] & =\frac{f_{n}^{*}\left(\mu_{c}\right)}{1-f_{h}^{*}\left(\mu_{c}\right)} \\
& =\frac{1}{\mu_{c} E\left[T_{h}\right]} .
\end{aligned}
$$

Fig. 11 shows the average number of handovers per call for the reference cell described in Section VI. This figure compares the results obtained from (27) and (36). Agreement of the two results is a justification of the validity of the proposed cell residence time distributions.

\section{Channel Holding Time Distribution}

The channel holding (or occupancy) time is a random variable defined as the length of time starting from the instant a channel in a cell is seized by the arrival of either a new or a handover call, until the time the channel is released either by

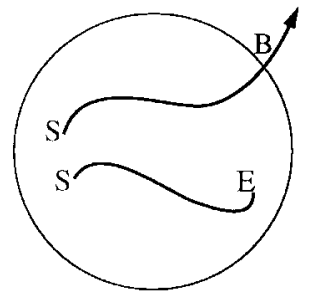

$S B=T_{n} \quad$ New call cell residence time $S E \equiv T \quad$ Call holding time

(a)

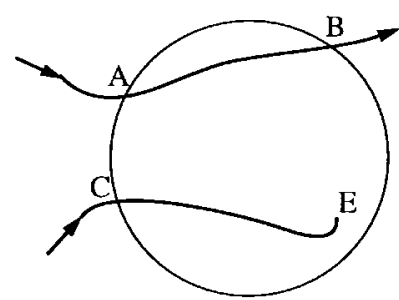

$$
\begin{array}{ll}
A B \equiv T_{h} & \text { Handover call cell residence time } \\
C E \equiv T_{c} & \text { Residual call time }
\end{array}
$$

(b)

Fig. 13. Illustration of the new and handover call cell residence time.

completion of the call or by handing over to another cell. In other words, the time spent by a user on a particular channel in a given cell is the channel holding time. The channel holding time is somewhat equivalent to the call duration in the fixed telephone network. However, in the case of cellular mobile networks, most often it only corresponds to a portion of the 


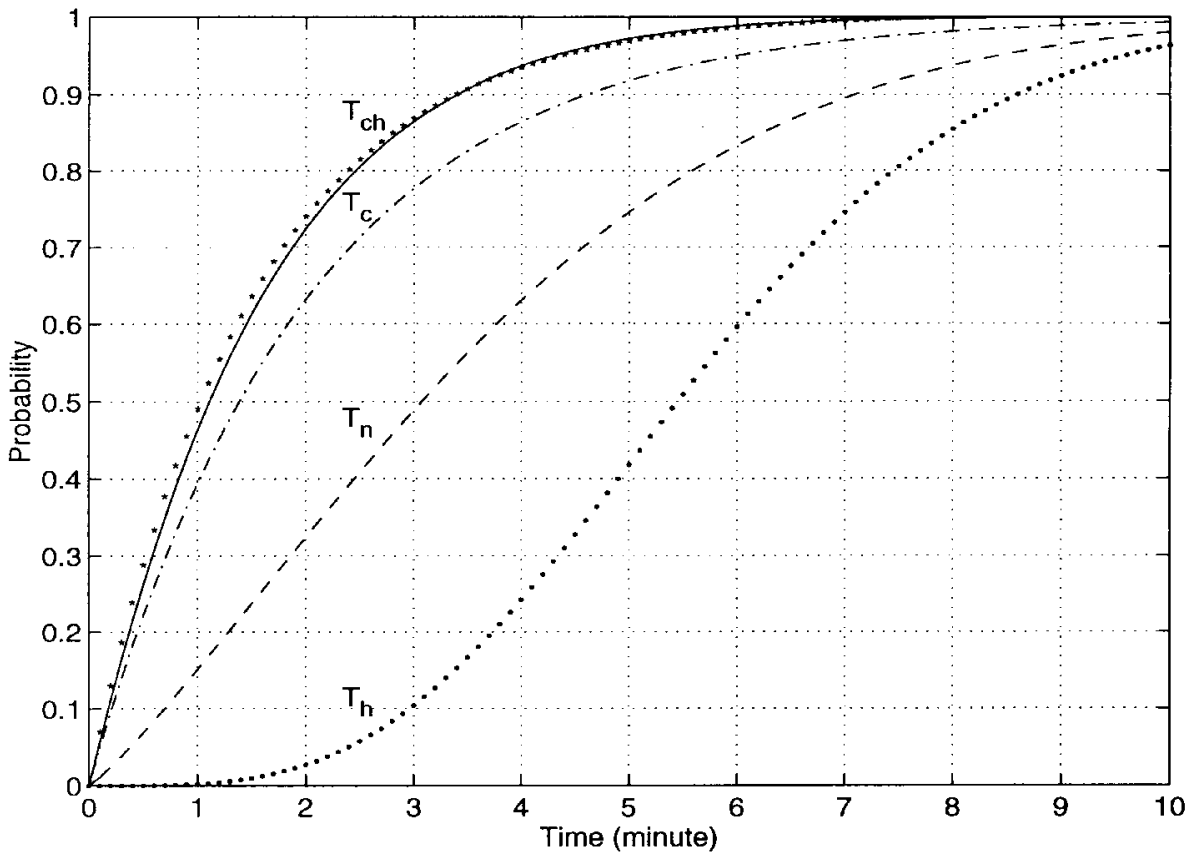

Fig. 14. Different distribution functions for a cell size of $3 \mathrm{~km}$.

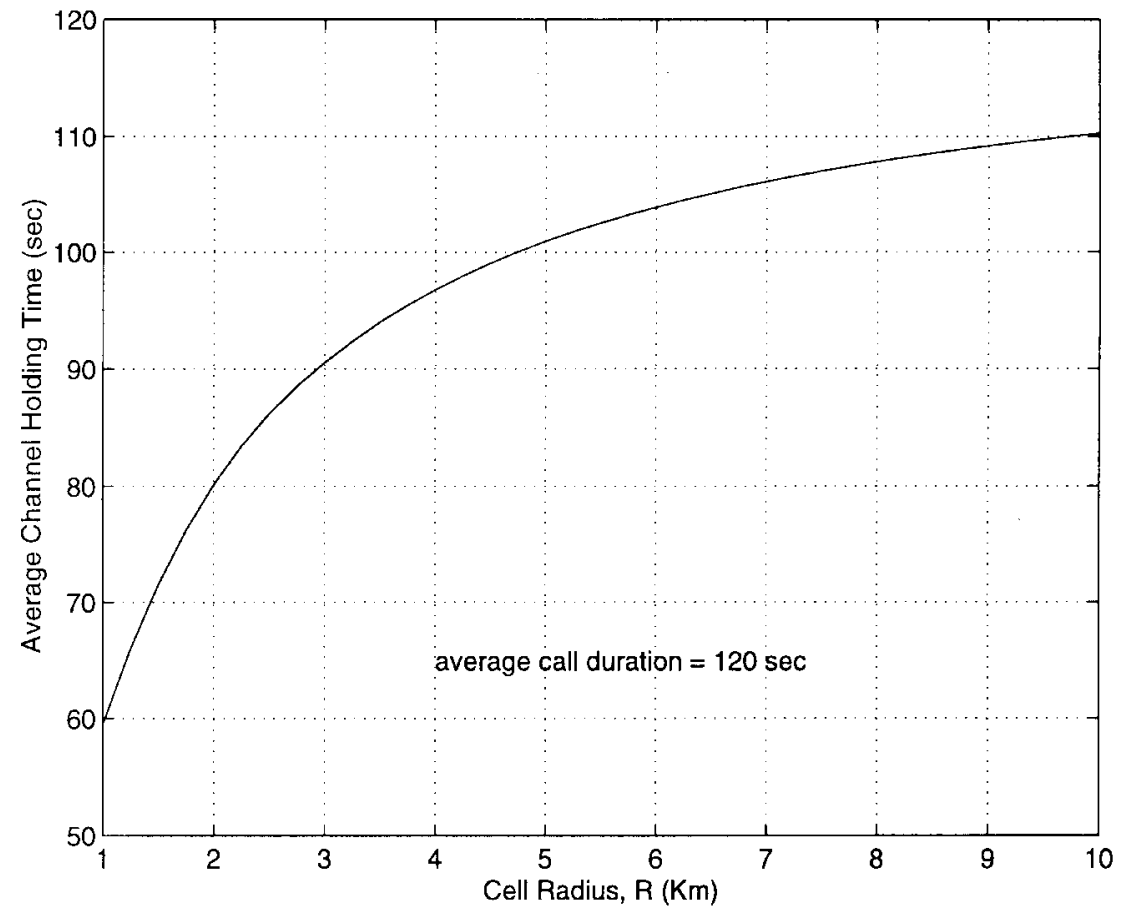

Fig. 15. Variation of the average channel holding time with cell size in reference cell.

total call duration in which the mobile is locatd in an associated cell.

The channel holding time is a function of the system parameters such as cell size, user location, user mobility, and call duration. In Fig. 12, the time intervals among points $\left(A_{1}, H_{11}\right),\left(A_{2}, C_{2}\right)$, and $\left(A_{3}, H_{31}\right)$ show the channel holding time for three new calls originating at points $A_{1}, A_{2}$, and $A_{3}$. The time intervals between points $\left(H_{11}, C_{1}\right),\left(H_{31}, H_{32}\right)$, and $\left(H_{32}, C_{3}\right)$ show the channel holding time for handover calls. When a new call is set up, a channel is occupied until the call is completed in the originating cell or the mobile moves out of the cell. Therefore, the channel holding time of the new call $T_{N}$ is either $T_{n}$ or $T_{C}$, whichever is less [Fig. 13(a)]

$$
T_{n}=\min \left(T_{n}, T_{c}\right) \text {. }
$$

A similar reasoning applies to a call which is handed over from a neighboring cell. In this case, the channel is occupied until the call is completed $[C E$ in Fig. 13(b)] or the mobile moves out to another cell $[A B$ in Fig. 13(b)]. Because of the memoryless property of the exponential distribution, the 
residual call time after a handover is independent of the time elapsed since the start of the call. As a result, the probability distribution of the residual call time given the time elapsed since the start of the call is the same as the original call duration $T_{c}$. Therefore, the channel holding time of the handover call $T_{H}$ is either $T_{h}$ or $T_{c}$, whichever is less

$$
T_{H}=\min \left(T_{h}, T_{c}\right)
$$

Since $T_{n}$ and $T_{h}$ are mainly dependent on the physical movement of the mobile, and have no influence on the total call duration $T_{c}$, it is reasonable to assume that the random variables $T_{n}$ and $T_{h}$ are independent of $T_{c}$. Therefore, the distribution function of the $T_{N}$ and $T_{H}$ can be calculated by

$$
\begin{aligned}
& F_{T_{N}}(t)=F_{T_{c}}(t)+F_{T_{n}}(t)-F_{T_{c}}(t) F_{T_{n}}(t) \\
& F_{T_{H}}(t)=F_{T_{c}}(t)+F_{T_{h}(t)}-F_{T_{c}}(t) F_{T_{h}}(t) .
\end{aligned}
$$

The distribution of the channel holding time in a given cell is a weighted function of $F_{T_{N}}(t)$ and $T_{T_{H}}(t)$. If $\zeta$ is the fraction of the average nonblocked new calls out of the average total number of calls in a cell, the fraction of the average number of successful handed over calls will be $1-\zeta$. Therefore, the distribution function of the channel holding time including both new and handover calls will be

$$
F_{T_{c h}}(t)=\zeta F_{T_{N}}(t)+(1-\zeta) F_{T_{H}}(t) .
$$

$\zeta$ can be expressed in terms of the average number of handovers per call $E[H]$ as

$$
\zeta=\frac{1}{1+E[H]} .
$$

Equation (40) can be rewritten in terms of the cell residence time and the call holding time distributions as

$$
\begin{gathered}
F_{T_{\mathrm{ch}}}(t)=F_{T_{c}}(t)+\frac{1}{1+E[H]}\left(1-F_{T_{c}}(t)\right) \\
\cdot\left(F_{T_{n}}(t)+E[H] F_{T_{h}}(t)\right) .
\end{gathered}
$$

A numerical solution to (42), assuming $T_{n}$ and $T_{h}$ with a generalized gamma distribution, indicates that the distribution function of the channel holding time in a cell follows an exponential distribution. Fig. 14 shows the distribution functions of the random variables $T_{n}, T_{h}, T_{c}$, and $T_{c h}$ for a cell size of $3 \mathrm{~km}$. The same figure shows a comparison of the exponential distribution with the same average value as that of $T_{c h}$. It can be seen that the channel holding time distribution fits well with the exponential distribution. This agrees with the result obtained in [3] and assumed in [1]. The average channel holding time is a function of cell parameters such as mobility, cell size, and average call holding time. Fig. 15 illustrates the variation of the average channel holding time with cell size for the reference cell. It shows that as the cell size increases, the average channel holding time $\overline{T_{c h}}$ approaches the average call holding time $\overline{T_{c}}$, which can be expected.

\section{CONCLusions}

In this paper, a mathematical formulation is developed for the systematic tracking of the random movement of a mobile station in a cellular environment. It incorporates mobility parameters under genrealized conditions in a quasirandom fashion with assigned degrees of freedom, so that the model could be tailored to be applicable in most cellular systems. The proposed model is used to characterize different mobilityrelated traffic parameters in cellular mobile communication systems. These include the distribution of the cell residence time of both new and handover calls, the channel holding time, and the average number of handovers per call. Results show that the generalized gamma distribution is adequate to describe the cell residence time distribution of both new and handover calls. It is also shown that the negative exponential distribution is a good approximation for the channel holding time distribution in cellular mobile systems. In order that the results could be made applicable to a wide range of cellular environments, it was shown that an increase in mobile drift in a cell can be treated as contributing to an effective increase in the cell radius. Similarly, it was shown that an increase in the speed of a mobile in a cell can be treated as contributing to a decrease in the cell size, and vice versa. Taking this excess cell radius into account for different values of drift and speed, a broad variety of cell coverage areas with different street orientations and traffic flows can be handled by the proposed mobility model.

\section{REFERENCES}

[1] D. Hong and S. S. Rappaport, "Traffic model and performance analysis for cellular mobile radio telephone systems with prioritized and nonprioritized handoff procedures," IEEE Trans. Veh. Technol., vol. VT-35, pp. 77-92, Aug. 1986.

[2] E. Del Re, R. Fantacci, and G. Giambene, "Handover and dynamic channel allocation techniques in mobile cellular networks," IEEE Trans. Veh. Technol., vol. 44, pp. 229-237, May 1995.

[3] R. A. Guerin, "Channel occupancy time distribution in a cellular radio system," IEEE Trans. Veh. Technol., vol. VT-35, pp. 89-99, Aug. 1987.

[4] S. Tekinay, "Modeling and analysis of cellular networks with highly mobile heterogeneous traffic sources," Ph.D. dissertation, George Mason Univ., Fairfax, VA, 1994.

[5] X. Luo and D. Everitt, "Handoff effects in microcellular systems," in Proc. 42nd IEEE Veh. Technol. Conf. (VTC'92), Denver, CO, May 1992, pp. 654-657.

[6] M. Frodigh, "Performance bounds for power control supported DCAalgorithms in highway micro cellular radio systems," IEEE Trans. Veh. Technol., vol. 44, pp. 238-243, May 1995.

[7] G. Morales-Andreas and M. Villen-Altamirano, "An approach to modeling subscriber mobility in cellular radio networks," in 5th World Telecommun. Forum, Geneva, Switzerland, Nov. 1987, pp. 185-189.

[8] R. Thomas, H. Gilbert, and G. Mazziotto, "Influence of the movement ove the mobile station on the performance of a radio cellular network," presented at the 3rd Nordic Seminar, Copenhagen, Denmark, paper 9.4, Sept. 1988.

[9] G. P. Pollini and D. J. Goodman, "Signalling system performance evaluation for personal communications," IEEE Trans. Veh. Technol., vol. 45, pp. 131-138, Feb. 1996.

[10] I. Seskar, S. Maric, J. Holtzman, and J. Wasserman, "Rate of location area updates in cellular systems," 42nd IEEE Veh. Technol. Conf. (VTC'92), Denver, CO, May 1992, pp. 694-697.

[11] S. Nanda, "Teletraffic models for urban and suburban microcells: Cell sizes and handoff rates," IEEE Trans. Veh. Technol., vol. 42, pp. 673-682, Nov. 1993.

[12] M. Inoue, H. Morikawa, and M. Mizumachi, "Performance analysis of microcellular mobile communication systems," in Proc. 44th IEEE Veh. Technol. Conf. (VTC'94), Stockholm, Sweden, June 1994, pp. 135-139. 
[13] K. L. Yeung and S. Nanda, "Optimal mobile-determined micro-macro cell selection," in Proc. 6th IEEE Int. Symp. Personal, Indoor and Mobile RAdio Commun. (PIMRC'95), Toronto, Canada, Sept. 1995, pp. 294-299.

[14] _ "Channel management in microcell/macrocell cellular radio systems," IEEE Trans. Veh. Technol., vol. 45, pp. 601-612, Nov. 1996.

[15] H. Xie and S. Kuek, "Priority handoff analysis," in Proc. 43rd IEEE Veh. Technol. Conf. (VTC'93), NJ, May 1993, pp. 855-858.

[16] H. Xie and D. J. Goodman, "Mobility models and biased sampling problem," in Proc. 2nd IEEE Int. Conf. Universal Personal Commun. (ICUPC'93), Ottawa, Canada, Oct. 1993, pp. 804-807.

[17] J. H. Sanchez Vargas, "Teletraffic performance of cellular mobile radio systems," Ph.D. disseration, Univ. Essex, England, 1988.

[18] X. Luo, "Investigation of traffic performance in mobile cellular communication systems," Master's thesis, Univ. Melbourne, Australia, 1991.

[19] Y. B. Lin, S. Mohan, and A. Noerpel, "Queueing priority channel assignment strategies for PCS handoff and initial access," IEEE Trans. Veh. Technol., vol. 43, pp. 704-712, Aug. 1994.

[20] A. D. Malyan, L. J. Ng, V. C. M. Leung, and R. W. Donaldson, "Network architecture and signalling for wireless personal communications," IEEE J. Select. Areas Commun., vol. 11, pp. 830-841, Aug. 1993.

[21] H. Jiang and S. Rappaport, "Handoff analysis for CBWL schemes in cellular communications," in Proc. 3rd IEEE Int. Conf. Universal Personal Commun. (ICUPC'94), San Diego, CA, 1994, pp. 496-500.

[22] W. M. Jolley and R. E. Warfield, "Modeling and analysis of layered cellular mobile networks," in Proc. 13th Int. Teletraffic Congr. (ITC-13), Copenhagen, Denmark, June 1991, pp. 161-166.

[23] M. Naghshineh and A. S. Acampora, "Design and control of microcellular networks with QOS provisioning for real time traffic," in Proc 3rd IEEE Int. Conf. Universal Personal Commun. (ICUP'94), San Diego, CA, Sept. 1994, pp. 376-381.

[24] F. N. Pavlidou, "Two-dimensional traffic models for cellular mobile systems," IEEE Trans. Commun., vol. 42, pp. 1505-1511, Feb./Mar./Apr. 1994.

[25] S. S. Rappaport and C. Purzynski, "Prioritized resource assignment for mobile cellular communication systems with mixed services and platform types," IEEE Trans. Veh. Technol., vol. 45, pp. 443-458, Aug. 1996.

[26] C. Purzynski and S. S. Rappaport, "Traffic performance analysis for cellular communication systems with mixed platform types and queued hand-offs," in Proc. 43rd IEEE Veh. Technol. Conf. (VTC'93), NJ, May 1993, pp. 172-175.

[27] S. S. Rappaport, "The multiple-call handoff problem in high capacity cellular communications systems," IEEE Trans. Veh. Technol., vol. 40, pp. 546-557, Aug. 1993.

[28] S. Rappaport and L. R. Hu, "Microcellular communication systems with hierarchical macrocell overlays: Traffic performance models and analysis," Proc. IEEE, vol. 82, pp. 1383-1397, Sept. 1994.

[29] D. C. Cox and D. O. Reudink, "Dynamic channel assignment in highcapacity mobile communications systems," Bell Syst. Tech. J., vol. 50, pp. 1833-1857, July 1971.

[30] _ "Increasing channel occupancy in large scale mobile radio systems: Dynamic channel reassignment," IEEE Trans. Commun., vol. COM-21, pp. 1302-1306, Nov. 1973
[31] L. Schiff, "Traffic capacity of three types of common-user mobile radio communication systems," IEEE Trans. Commun. Technol., vol. COM-18, no. 1, pp. 12-21, Feb. 1970.

[32] M. M. Zonoozi and P. Dassanayake, "A novel modeling technique for tracing mobile users in a cellular mobile communication system," Int. J. Wireless Personal Commun., accepted for publication.

[33] S. S. Rappaport, "Blocking, handoff and traffic performance for cellular communication systems with mixed platforms," Proc. Inst. Elect. Eng., vol. 140 , pt. I, pp. 389-401, Oct. 1993

[34] W. C. Wong, "Packet reservation multiple access in a metropolitan microcellular radio environment," IEEE J. Select. Areas Commun., vol. 11, pp. 918-925, Aug. 1993.

[35] D. R. Cox and P. A. W. Lewis, The Statistical Analysis of Series of Events. London: Chapman and Hall, 1978.

[36] A. M. Law and W. D. Kelton, Simulation Modeling and Analysis. New York: McGraw-Hill, 1991.

[37] G. A. Mihram, Simulation, Statistical Foundations and Methodology. New York: Academic, 1972.

[38] S. M. Ross, Stochastic Process. New York: Wiley, 1983.

[39] L. Kleinrock, Queueing Systems, Volume 1: Theory. New York: Wiley, 1975 .

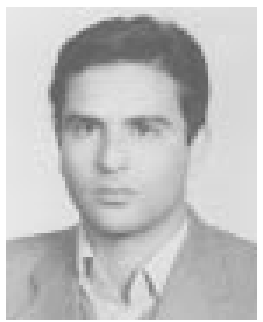

Mahmood M. Zonoozi received the B.Sc. and M.Sc. degrees in electrical engineering from K. N. Toosi University, Iran.

He has worked in the Satellite and Microwave Communication Departments of Iran Telecom Company for several years. He is currently working toward the Ph.D. degree at the Victoria University of Technology, Melbourne, Australia. He is attached to the Mobile Communications and Signal Processing Research Group of the University, and his project deals with the issues involved in handover and teletraffic of cellular mobile communication systems.

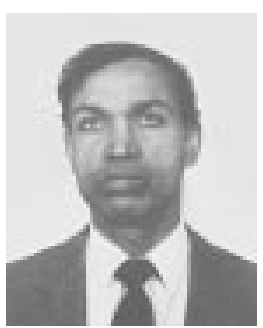

Prem Dassanayake (M'83) received the B.Sc.Eng. degree from the University of Sri Lanka, and the M.Sc. and Ph.D. degrees from the University of Wales, Cardiff, U.K

At present, he is with the Department of Electronics and Electrical Engineering, Victoria University of Technology, Footscray, Australia. Prior to joining Victoria University, he worked at the University of Moratuwa, Sri Lanka, and the University of Bahrain Bahrain. He has also been a Visiting Researcher at the Bureau of Medical Devices, Health and Welfare, Ottawa, Canada, and at Telstra Research Laboratories, Clayton, Australia. 